
\title{
28 Research Square \\ Gut microbiota signatures in Schistosoma japonicum infection-induced liver cirrhosis patients
}

\section{Qifeng Gui}

Zhejiang University School of Medicine First Affiliated Hospital

\section{Feng Zhu}

Zhejiang University School of Medicine First Affiliated Hospital

\section{Chi Xiao}

Hangzhou Medical College

\section{Haifeng Lu}

Zhejiang University School of Medicine First Affiliated Hospital

\section{Qin Zhang}

Zhejiang University School of Medicine

Jia Xu

Zhejiang University School of Medicine First Affiliated Hospital

Huilin Jin

Wangdian People's Hospital, Jiaxing, Zhejiang

Yunmei Yang (D1194070@zju.edu.cn )

The First Affiliated Hospital, School of Medicine, Zhejiang University

\section{Research}

Keywords: Gut microbiota, 16S Rrna, Schistosoma japonicum

Posted Date: September 1st, 2020

DOI: https://doi.org/10.21203/rs.3.rs-68447/v1

License: (c) (i) This work is licensed under a Creative Commons Attribution 4.0 International License.

Read Full License 


\section{Abstract}

Background: One of the serious complications induced by Schistosoma japonicum infection is the development of liver cirrhosis. Several studies have assessed the role of gut microbiota in cirrhosis of different etiologies, but none has done so in the context of $S$. japonicum infection in humans. Here, to explore the possible role of gut microbiota in $S$. japonicum infection-induced liver cirrhosis, we conducted an observational clinical study.

Methods: Twenty-four patients with S. japonicum infection-induced liver cirrhosis and 25 age- and gender-matched controls from Zhejiang Province, China, were enrolled in the study. Fecal samples were collected and used for 16S rRNA gene hypervariable V4 region Illumina MiSeq sequencing.

Results: Eight hundred seven operational taxonomic units (OTUs) were detected, of which 491 were common in the two groups, while 123 and 193 were unique for the control and cirrhosis groups, respectively. Overall, no significant differences in both alpha and beta diversities and overall microbiota structure were found in the two groups. However, Bacilli (Class) and Lactobacillales (Order) were significantly higher in patients with $S$. japonicum infection-induced liver cirrhosis than in healthy individuals.

Conclusions: Altogether, our results suggest that the gut microbiota of $S$. japonicum infection-induced liver cirrhosis patients is similar to that of healthy individuals.

\section{Background}

Schistosomiasis is one of the most prevalent parasitic diseases in the world [1]. An estimated 240 million people are affected by this disease, together with 800 million more at risk of infection [2]. Worryingly, schistosomiasis alone is associated with a high disease burden of as much as 3.3 million disabilityadjusted life years (DALYs), accounting for more than $10 \%$ of the global burden of Neglected Tropical Diseases (NTDs) [3, 4]. There are 5 known etiologic agents of schistosomiasis, all of which are trematode parasites of the Schistosoma genus: S. japonicum, S. mansoni, S. haematobium, S. intercalatum, and S. mekongi [5]. Only S. japonicum exists in China, and it is predominant in 12 provinces south of the Yangtze River [6]. In the 1950s, an estimated 11.6 million people were infected, while more than 100 million more were at risk of infection [7]. From the mid-1950s, the number of infected cases has reduced by over $99 \%$; however, schistosomiasis is still endemic in 140 counties in China [1].

Liver cirrhosis is a common (end-stage) consequence of several liver diseases and a major cause of mortality worldwide [8]. Previous studies have reported an association between gut microbiota alterations and chronic liver disease $[9,10]$, including cirrhosis secondary to hepatitis $B$ or $C$ virus infections $[11,12]$, alcohol over-consumption [13, 14], nonalcoholic fatty liver disease [13, 15, 16], and even primary biliary cirrhosis [17]. Furthermore, cirrhosis complications, including hepatic encephalopathy, spontaneous bacterial peritonitis, and sepsis, were linked to gut microbiota alterations [18]. Some studies on the gut- 
liver axis have even demonstrated that the gut microbiota (and bacterial translocation) plays an important role in the pathogenesis of cirrhosis and its complications $[13,19]$.

Recent studies using not only animal models but also human clinical samples have demonstrated a clear relationship between gut microbiota alterations and Schistosoma spp. infection [20, 21, 22, 23, 24, 25]. However, no study has explored the relationship between gut microbiota and S. japonicum infectioninduced liver cirrhosis; the potential role of gut microbiota alterations in disease onset and progression is still unclear.

Here, to explore the possible association between gut microbiota and S. japonicum infection-induced liver cirrhosis, we conducted an observational clinical study. Fecal samples collected from 24 patients and 25 matched healthy individuals from the Zhejiang Province, China, were used for 16S rRNA Illumina MiSeq sequencing. The main objectives of this study were: (1) to characterize the relevant gut microbiota alterations associated with $S$. japonicum infection-induced liver cirrhosis; and (2) to identify potential bacterial taxa to be used as non-invasive biomarkers for the early diagnosis of liver cirrhosis secondary to schistosomiasis.

\section{Methods}

\section{Ethics Statement}

This study was conducted according to the principles expressed in the Declaration of Helsinki. All experiments were approved by the First Affiliated Hospital Clinical Research Ethics Committee, School of Medicine, Zhejiang University (ref: 2017411-1), and the Jiaxing Wangdian People's Hospital Ethics Committee (ref: 2017002). All participants provided written informed consent.

\section{Exclusion criteria and subject enrolment}

Eligibility was defined by the application of the following exclusion criteria: (I) history of digestive disorders, such as other causes of liver disease, irritable bowel syndrome, inflammatory bowel disease, and chronic diarrhea; (II) history of previous gastrointestinal surgery; (III) history of probiotics, prebiotics, synbiotics, antibiotics, acid suppressors, metformin, and gastrointestinal motility targeting-drugs use, less than eight weeks before enrollment; (IV) history of bowel preparation, less than four weeks before enrollment; (V) diagnosis of major diseases such as malignant tumors, cardiovascular disease, respiratory, autoimmune and allergic diseases, neurological disorders, renal insufficiency, diabetes, uncontrolled hypertension (blood pressure $\geq 150$ / $90 \mathrm{mmHg}$ ), dyslipidemia, depression, mania, and bipolar affective disorder; and (VI) hepatitis B virus, hepatitis $\mathrm{C}$ virus and HIV infection.

From December 2017 to November 2019, 24 patients with S. japonicum infection-induced liver cirrhosis and 26 age- and gender-matched healthy individuals were enrolled from Hangzhou and Jiaxing, northern cities of the Zhejiang Province, China, an S. japonicum infection endemic area. Since the stool specimen from one healthy individual was unsatisfactory, the healthy controls group was ultimately composed of 
25 individuals. All participant demographics information, laboratory tests, computed tomography (CT), and B ultrasonic scans were collected from the hospital electronic medical records system. Additionally, questionnaires were filled by all subjects. Schistosomiasis diagnosis was performed using the Kato-Katz method to detect $S$. japonicum eggs in stool samples. Liver cirrhosis was diagnosed according to clinical and biochemical data and imaging exams (CT or B ultrasonic scans) [26, 27]. Liver function was evaluated with the Child-Pugh classification system [28].

\section{Fecal sample collection}

The participants were trained by a researcher to obtain a complete stool collection method. Fresh stool samples (self-collected from patients and controls) were collected into anaerobic bags (Mitsubishi Gas Chemical). Samples were divided into five $200 \mathrm{mg}$ aliquots, in the first $30 \mathrm{~min}$ after collection, and immediately stored at $-80^{\circ} \mathrm{C}$ for posterior analysis.

\section{Genomic DNA extraction}

Total genomic DNA was extracted per sample using the MagPure Stool DNA KF kit B (Magen,Guangzhou, China) according to the manufacturer's instructions. DNAs were quantified in the Qubit Fluorometer (Invitrogen, Qubit 2.0) using the Qubit® dsDNA BR Assay kit (Invitrogen, CA, USA). DNA quality was assessed using gel electrophoresis (1\% agarose gels). Negative controls containing the elution buffer only were included during DNA extraction and quantification to monitor possible contamination.

\section{Library construction and sequencing}

Bacterial 16S rRNA gene hypervariable region V4 was amplified using degenerate polymerase chain reaction (PCR) and the primers 515Fw (5'-GTGCCAGCMGCCGCGGTAA-3') and 806Rv (5'GGACTACHVGGGTWTCTAAT-3'), which contained the Illumina adapter sequence, a pad, and a linker. Fifty microliters reaction volumes, containing $30 \mathrm{ng}$ of DNA templates together with the primers $(10 \mu \mathrm{M})$ and the PCR master mix, were used. Amplification was performed under the following conditions: $95^{\circ} \mathrm{C}$ for 3 minutes; 30 cycles of $95^{\circ} \mathrm{C}$ for 45 seconds, $56^{\circ} \mathrm{C}$ for 45 seconds, and $72^{\circ} \mathrm{C}$ for 45 seconds; and a final extension step at $72^{\circ} \mathrm{C}$ for 10 minutes. PCR products were purified using Agencourt AMPure XP beads (Beckman Coulter, CA, USA) according to the manufacturer's instructions. Library quality was evaluated using the 2100 Bioanalyzer (HT DNA 1K Labchip 760517) instrument (Agilent Technologies, CA, USA). Sequencing was performed using the Illumina HiSeq 2500 platform (BGI, Shenzhen, China) following the Illumina standard pipelines to get $2 \times 250$ bp paired-end reads.

\section{Bioinformatics analysis}

Raw reads were filtered to remove adaptors and low-quality and/or ambiguous bases, and paired-end reads were merged using the Fast Length Adjustment of Short reads (FLASH, v1.2.11) software [29]. Sequences were clustered into operational taxonomic units (OTUs) with a cutoff value of $97 \%$ using the UPARSE software (v7 .0.1090) [30]. Chimeric sequences were detected (compared with the Gold database) using the UCHIME software (v4.2.40) [31] and removed from the analysis. Sequences of representative OTUs were then taxonomically classified using the Ribosomal Database Project Classifier 
(v.2.2) with a minimum confidence threshold of 0.6 and trained on the Greengenes database (v201305) using the QIIME pipeline (v1.8.0) [32]. The USEARCH_global command [33] was used to quantify OTUs abundance in each sample.

\section{Bacterial diversity analysis}

Alpha and beta diversities were estimated using the MOTHUR software (v1.31.2) [34] and the QIIME pipeline (v1.8.0) [32], respectively. Sample clustering was conducted using the QIIME pipeline (v1.8.0) [32] and the unweighted pairwise grouping method with averaging (UPGMA). Barplots and heatmaps of different classification levels were plotted with the R package (v3.4.1) and the R package "gplots" tool, respectively. Venn diagrams and accumulation curves were obtained using the R package "VennDiagram" (v 3.1.1). A principal component analysis (PCA) was performed using the R package "ade4" tool. Taxonomic characterization was performed using a Linear Discriminant Analysis (LDA) with the calculation of the LDA effect size (LEfSe).

\section{Statistical analysis}

Due to the lack of data on effect size, a power calculation might be difficult. However, a recent similar study on S. japonicum infection in which each group consisted of 24 animal subjects might have helped with power calculations [23]. Continuous variables are presented as mean \pm standard deviation (SD), and categorical variables are presented as percentages (\%). The student's $t$-test or Wilcoxon test and the $\chi 2$ test were used to evaluate differences between the two groups for continuous and categorical variables, respectively. Whenever applicable, the false discovery rate (FDR) was used to correct the calculated $p$ values. Statistical analyses were performed using the SPSS software (v 22.0; SPSS, IL, USA). Statistical significance was given by $\mathrm{p}<0.05$, FDR $<0.05$, or LDA scores $>2$.

\section{Results}

\section{Participants' characterization}

The study population included a total of 24 patients with S. japonicum infection-induced liver cirrhosis (12 males and 12 females), and 25 age- and gender-matched healthy individuals (12 males and 13 females). The average ages of cirrhosis and control groups were comparable: $82.7 \pm 7.3$ years old and $82.3 \pm 7.1$ years old, respectively (Table 1 ). Furthermore, no significant differences were observed concerning gender, body mass index (BMI), or smoking and drinking habits between the two groups. In addition, the platelet counts and the serum levels of total bilirubin (TB), direct bilirubin (DB), and alanine aminotransferase (ALT) were comparable between the groups. On the other hand, while the serum levels of aspartate aminotransferase (AST) and glutamyl transpeptidase (GGT), together with the prothrombin time (PT) were significantly increased in the S. japonicum infection-induced liver cirrhosis group, the total protein, and globulin serum levels were significantly decreased in the same group, always in comparison with the control group (Table 1 ; at least $\mathrm{p}<0.05$ ). Regarding disease severity stratification, performed only 
for the cirrhosis group, 13 patients (54.2\%) were classified as Child-Pugh A, 7 (29.2\%) as Child-Pugh B, and $4(16.7 \%)$ as Child-Pugh C (Table 1$)$.

\section{Gut microbial diversity is comparable in individuals with $\mathrm{S}$. japonicum infection-induced liver cirrhosis and healthy controls}

To characterize the gut microbiota in the context of S. japonicum infection-induced liver cirrhosis, we sequenced bacterial $16 \mathrm{~S}$ rRNAs extracted from fecal samples and employed an OTU-based bioinformatics analysis to estimate alpha and beta diversities. Importantly, the obtained accumulation curve reached a plateau, indicating that most of the gut microbial populations have been detected at the used sequencing depth (Fig. 1). A total of 807 OTUs were obtained, of which 491 were equally detected in samples from cirrhosis individuals and healthy controls (Fig. 2). Notably, as represented in the Venn diagram, 123 and 193 OTUs were uniquely detected in healthy individuals and patients with S. japonicum infection-induced liver cirrhosis, respectively (Fig. 2).

Concerning alpha diversity, we used 6 different metrics for the analysis: the Observed species, Chao, ACE, Shannon, Simpson, and Good's coverage indexes were calculated. Overall, we did not detect any significant changes when healthy individuals and patients with $S$. japonicum infection-induced liver cirrhosis were compared. However, a slight tendency towards a lower bacterial diversity was observed in the cirrhosis group (particularly looking at Observed species, Chao, ACE, and Shannon indexes) (Fig. 3). In addition, considering the small number of cases showing the disease severity in the cirrhosis group (13 Child-Pugh A, 7 Child-Pugh B, and 4 Child-Pugh $C$ cases), we did not discuss the variance in microbiota data further by disease severity.

We further analyzed beta diversity (based on weighted UniFrac distances), to evaluate differences between samples. Overall, and once more, no major differences were observed when healthy individuals and patients with S. japonicum infection-induced liver cirrhosis were compared (Fig. 4A). PCA data (Fig. 4B) confirmed this similarity. Samples from each group showed heterogeneous distributions that were not species-specific, indicating that the structure of gut bacterial communities is similar in healthy individuals and patients with S. japonicum infection-induced liver cirrhosis (Fig. 4B).

\section{Fecal microbial communities of healthy individuals and patients with S. japonicum infection-induced liver cirrhosis are slightly different}

Next, we employed a qualitative approach to perform a more in-depth characterization of the intestinal bacterial communities present in the stool samples collected from patients and controls. Briefly, we evaluated bacterial relative abundance at both phylum and genus levels (Fig. 5A and B, respectively). The 
analysis of the most abundant bacterial phyla in the fecal samples collected from the two different groups of individuals revealed a similar picture: Bacteroidetes, Firmicutes, and Proteobacteria were the three most relevant phyla in both groups, accounting for $97.1 \%$ and $94.4 \%$ frequencies in healthy controls and cirrhosis patients, respectively (Fig. 5A). On the other hand, some different patterns were observed at the genus level, being Bacteroides, Prevotella, and Faecalibacterium, the three most prevalent genera in the gut of healthy individuals, while in cirrhosis patients, the third most prevalent genus was Escherichia (Fig. 5B).

Additionally, to explore statistical relevance, we performed a LEfSe analysis: $p<0.05$ (Wilcoxon test) and an LDA scores $>2$ were considered statistically significant (Figs. 6 and 7). Of note, we also considered FDR values in this analysis to minimize the number of false-positive results. Overall, we have found that only the Bacilli Class and the Lactobacillales Order were significantly increased in cirrhosis patients compared to healthy individuals (Fig. 8).

\section{Discussion}

One of the serious complications induced by S. japonicum infection is the development of liver cirrhosis [35]. Most egg-laying adult Schistosoma trematodes have liver tropism, and the local (hepatic) host immune responses to the deposited eggs result in granuloma formation, which eventually evolves to progressive liver fibrosis and portal hypertension [36]. Both in vivo and clinical studies focusing on $S$. japonicum infections revealed that hepatic stellate cells (HSCs) are the key drivers of hepatic fibrosis [35, 37], via collagen production and deposition. Importantly, liver fibrosis presentations have the potential to evolve into liver cirrhosis or liver cancer in the future [38]. Several studies have assessed the role of gut microbiota in cirrhosis of different etiologies [11, 16, 39, 40,41]. Microbiota dysbiosis was often reported in cirrhosis patients: (I) autochthonous/non-autochthonous taxa ratio reduction; (II)

Firmicutes/Bacteroidetes ratio inversion; or (III) increased prevalence of potentially pathogenic bacteria $[42,43,44]$. Interestingly, the liver-gut axis was involved in the physiopathology of cirrhosis: overgrown intestinal bacteria have the potential to translocate across the leaky gut barrier and reach the liver, leading to cirrhosis development or progression [45]. However, few studies explored the gut microbiota in the context of $S$. japonicum infection-induced liver cirrhosis.

In fact, to our knowledge, this is the first study exploring the possible association between gut microbiota and $S$. japonicum infection-induced liver cirrhosis in humans. Surprisingly, here, we found that the microbiota of S. japonicum infection-induced liver cirrhosis patients was as rich and diverse as that of matched healthy individuals. Overall, these results are not consistent with findings reported for in vivo studies. For instance, in a mouse model of $S$. japonicum Ova-induced granulomas, a significantly higher beta diversity was detected versus controls [23]. Similarly, an overall reduction in alpha and a significant increase in beta diversities were found in the gut of $S$. mansoni infected mice compared to uninfected controls [21]. However, some studies align with our findings. For instance, Schneeberger et al. suggested that $S$. mansoni infection (in children) does not affect the gut microbial composition [25]. 
Furthermore, the taxonomic composition at the phylum level was similar in cirrhosis patients and healthy individuals (prevalence of Bacteroidetes, Firmicutes, and Proteobacteria), aligning with S. japonicum in vivo infections' data [23]. Additionally, even at the genus level, no major changes were detected, indicating that in our context, there is no gut dysbiosis in S. japonicum infection-induced liver cirrhosis patients. This result is somehow unexpected and different from other studies that have reported gut dysbiosis in the context of human schistosomiasis (e.g., those caused by S. mansoni and S. haematobium) [20, 21, 22, 23, 24]. Additionally, in vivo studies have reported Schistosoma infection-related alterations in gut microbial composition. For instance, $S$. japonicum infection in mice was associated with significant changes in the gut prevalence of Firmicutes (relative decrease), Bacteroidetes, and Proteobacteria (relative increase) [23].

However, we did find some composition alterations in the gut microbiota of S. japonicum infectioninduced liver cirrhosis patients. Bacilli (at the class level) and Lactobacillales (at the order level) were significantly increased in patients with $S$. japonicum infection-induced liver cirrhosis compared to healthy individuals. Importantly, our results are consistent with those of a previous study, which may suggest that the increased prevalence of Lactobacillales in gut microbiota is a hallmark of human liver cirrhosis [46]. Additionally, a different study showed that Lactobacillales and Bacilli were the key contributors to gut dysbiosis in primary liver cancer and proposed them as potential diagnostic markers [47]. Altogether, these findings suggest a strong association between gut Bacilli and Lactobacillales and liver disease that deserves to be further explored in different pathological contexts.

Overall, our data do not support the idea that $S$. japonicum infection-induced cirrhosis is associated with significant alterations of gut microbiota profiles, opposing hypotheses proposed by others concerning schistosomiasis. Some reasons may justify our opposing report. Different studies focused on different Schistosoma spp. that have different organ tropisms: e.g., S. mansoni and S. japonicum target the gut and liver, while $S$. haematobium targets the bladder and the urogenital system [20, 21, 22, 24]. Importantly, the notion that $S$. haematobium infection was equally associated with human gut dysbiosis suggests that potential alterations of intestinal microbial communities in the context of schistosomiasis are probably a consequence of infection-induced systemic immune responses (and not of local events). In fact, some studies suggest this [20,21,22]. For instance, mesenteric lymph node cells isolated from mice infected with $S$. mansoni (dysbiotic) responded differently compared to the ones isolated from controls: higher interleukin (IL)-4, IL-10, and IL-17 secretion, together with lower interferon-y production [22]. Relevantly, aging is accompanied by a deterioration of immune responses, recently named as immunosenescence or immune-aging [48]. In our study, the enrolled patients were quite old (average of $82.7 \pm 7.3$ years old), which may justify the absence of phenotype: their potentially weaker immune responses to $S$. japonicum infection were not strong enough to impact their gut microbial communities.

Nevertheless, our geographic target-region conditioned the age range of our study population, which is a limitation of our study. Since 1995, due to the application of effective Schistosomiasis control measures, there are almost no newly reported cases in Zhejiang [1]. Therefore, patients with S. japonicum infectioninduced liver cirrhosis are not many and tend to be of advanced ages [27]. Therefore, we cannot exclude a 
possible age selection bias that, however, reflects the reality of the population studied: among the cirrhosis patients, 17 and 7 individuals were 80 or 90 years of age or older, respectively, and none of the others was less than 60 years old. We can, however, also hypothesize that our population has a survival bias. Living patients may be the ones with better outcomes due to stable gut microbiota profiles, while the fatalities may have been a consequence of gut microbiota disorders. This is, however, only a speculative exercise. Therefore, further studies focusing on much larger and heterogeneous populations (different geographies, with a wider age range) are needed to completely understand if $S$. japonicum infectioninduced liver cirrhosis is accompanied by gut microbiota alterations. In addition, the effective Schistosomiasis control in Zhejiang and advanced age of participants might affect our conclusion because it was generally almost impossible to include a cohort of age-matched acute $S$. japonicum infected patients without liver cirrhosis.

An additional factor that should be considered in future studies is the timing of infection, particularly if the study is designed to find biomarkers for cirrhosis diagnosis and/or prognosis. Indeed, the infection status may also condition the results. Human studies have mainly targeted adolescents [20] or children [24], and consequently, the early phase of infection. Additionally, the most commonly used mouse model of schistosomiasis in microbiome studies evaluated acute infection - less than 8 weeks post-challenge according to the model characterization $[49,50,51]$. The in vivo published data refers to stool samples collected at 28 days [21], 42 days [23], and 8 weeks [22] after infection. We cannot exclude that our different findings are just a consequence of our study context. Cirrhosis is the end-stage of chronic schistosomiasis, quite far time-wise from the early phase of infection. Both systemic immune responses and Schistosoma infection were associated with gut microbiota alterations [20]. However, in S. japonicum infection-induced liver cirrhosis, both are expected to decrease in magnitude, which may partially explain our findings.

\section{Conclusion}

In conclusion, overall, we showed that gut microbial taxonomic profiles and diversity in S. japonicum infection-induced liver cirrhosis patients appear to be similar to the ones from age-matched healthy people. Therefore, in our context, patients with S. japonicum infection-induced liver cirrhosis present a "healthy" gut structure, which may partially explain the better prognosis of cirrhosis secondary to this infection, compared to cirrhosis of different etiology in China [26, 27]. Future studies with much larger sample sizes across wider age ranges are, however, needed to further explore the relationship between $S$. japonicum infection-induced liver cirrhosis and gut microbiota and potentially disclose the possible mechanisms by which gut microbiota alterations influence cirrhosis and/or vice versa. Importantly, these studies should also evaluate immunity and inflammation to finally address if the gut-liver axis is implicated in S. japonicum infection-induced liver cirrhosis development and progression. Such studies should be further complemented with in vivo works with transplantation of human gut microbiota samples to ultimately establish (or exclude) a causal relationship between dysbiosis and cirrhosis secondary to schistosomiasis. 


\section{Declarations}

Acknowledgements

We would like to thank Editage (www.editage.cn) for English language editing, and BGI-Shenzhen for genome-sequencing services.

\section{Authors' contributions}

Conceptualization: Y.M.Y. and H.L.J. Data curation: Q.F.G. and F.Z. Formal analysis: Q.F.G., C.X. and J.X. Funding acquisition: Q.F.G., C.X. and Q.Z. Investigation: F.Z. Methodology: H.F.L. Supervision: Q.Z. Writing - original draft: Q.F.G. Writing - review \& editing: Y.M.Y. and H.L.J. All authors read and approved the final manuscript.

\section{Funding}

This study was supported by the Key Disciplines Construction Plan of Zhejiang Province Traditional Chinese Medicine (2017-XK-A31), the Chinese Medicine Research Program of Zhejiang Province (2020ZB148), and the National Key Research and Development Program of China (2018YFC2000301, 2018YFC2000500).

\section{Availability of data and materials}

The data supporting the conclusions of this article are included within the article. The datasets analysed during the present study are available from the corresponding author upon reasonable request.

\section{Ethics approval and consent to participate}

All participants were approved by the First Affiliated Hospital Clinical Research Ethics Committee, School of Medicine, Zhejiang University (ref: 2017411-1), and the Jiaxing Wangdian People's Hospital Ethics Committee (ref: 2017002). All participants provided written informed consent in Chinese. Participation was entirely voluntary and only participants with signed informed consent forms were enrolled into the study. and the rights of these subjects were protected.

\section{Consent for publication}

Not applicable.

\section{Competing interests}

The authors declare that they have no competing interests.

\section{Abbreviations}


OTUs: operational taxonomic units; DALYs: disability-adjusted life years; NTDs: Neglected Tropical Diseases; CT: computed tomography; PCA: principal component analysis; LDA: Linear Discriminant Analysis; LEfSe: LDA effect size; SD: standard deviation; FDR: false discovery rate; ALT: alanine aminotransferase; AST: spartate aminotransferase; BMI: body mass index; DB: direct bilirubin; GGT: glutamyl transpeptidase; PT: prothrombin time; SLC: S. japonicum infection-induced liver cirrhosis; TB: total bilirubin; HSCs: hepatic stellate cells; IL: interleukin.

\section{References}

1. Cao ZG, Zhao YE, Lee Willingham A, Wang TP. Towards the Elimination of Schistosomiasis japonica through Control of the Disease in Domestic Animals in The People's Republic of China: A Tale of over 60Years. Adv Parasitol. 2016;92:269-306.

2. Andrade G, Bertsch DJ, Gazzinelli A, King CH. Decline in infection-related morbidities following drugmediated reductions in the intensity of Schistosoma infection: A systematic review and metaanalysis. PLoS Negl Trop Dis. 2017;11 2:e0005372.

3. Steinmann P, Keiser J, Bos R, Tanner M, Utzinger J. Schistosomiasis and water resources development: systematic review, meta-analysis, and estimates of people at risk. Lancet Infect Dis. 2006;6 7:411-25.

4. Murray CJ, Vos T, Lozano R, Naghavi M, Flaxman AD, Michaud C, et al. Disability-adjusted life years (DALYs) for 291 diseases and injuries in 21 regions, 1990-2010: a systematic analysis for the Global Burden of Disease Study 2010. Lancet. 2012;380:9859:2197-223.

5. Chuah C, Gobert GN, Latif B, Heo CC, Leow CY. Schistosomiasis in Malaysia: A review. Acta Trop. 2019;190:137-43.

6. Zhang SQ, Sun CS, Wang M, Lin DD, Zhou XN, Wang TP. Epidemiological Features and Effectiveness of Schistosomiasis Control Programme in Lake and Marshland Region in The People's Republic of China. Adv Parasitol. 2016;92:39-71.

7. Li ZJ, Ge J, Dai JR, Wen LY, Lin DD, Madsen H, et al. Biology and Control of Snail Intermediate Host of Schistosoma japonicum in The People's Republic of China. Adv Parasitol. 2016;92:197-236.

8. Lozano R, Naghavi M, Foreman K, Lim S, Shibuya K, Aboyans V, et al. Global and regional mortality from 235 causes of death for 20 age groups in 1990 and 2010: a systematic analysis for the Global Burden of Disease Study 2010. Lancet. 2012;380:9859:2095-128.

9. Brenner DA, Paik YH, Schnabl B. Role of Gut Microbiota in Liver Disease. J Clin Gastroenterol. 2015;49(Suppl 1):25-7.

10. Wang F, Huang ZA, Chen X, Zhu Z, Wen Z, Zhao J, et al. LRLSHMDA: Laplacian Regularized Least Squares for Human Microbe-Disease Association prediction. Sci Rep. 2017;7 1:7601.

11. Heidrich B, Vital M, Plumeier I, Doscher N, Kahl S, Kirschner J, et al. Intestinal microbiota in patients with chronic hepatitis $C$ with and without cirrhosis compared with healthy controls. Liver Int. 2018;38(1):50-8. 
12. Ren Z, Li A, Jiang J, Zhou L, Yu Z, Lu H, et al. Gut microbiome analysis as a tool towards targeted non-invasive biomarkers for early hepatocellular carcinoma. Gut. 2019;68 6:1014-23.

13. Acharya C, Bajaj JS. Gut Microbiota and Complications of Liver Disease. Gastroenterol Clin North Am. 2017;46:1:155-69.

14. Starkel P, Schnabl B. Bidirectional Communication between Liver and Gut during Alcoholic Liver Disease. Semin Liver Dis. 2016;36 4:331-9.

15. Caussy C, Tripathi A, Humphrey G, Bassirian S, Singh S, Faulkner C, et al. A gut microbiome signature for cirrhosis due to nonalcoholic fatty liver disease. Nat Commun. 2019;10 1:1406.

16. Loomba R, Seguritan V, Li W, Long T, Klitgord N, Bhatt A, et al. Gut Microbiome-Based Metagenomic Signature for Non-invasive Detection of Advanced Fibrosis in Human Nonalcoholic Fatty Liver Disease. Cell Metab. 2017;25(5):1054-62.e5.

17. Quigley EM. Primary Biliary Cirrhosis and the Microbiome. Semin Liver Dis. 2016;36:4:349-53.

18. Lachar J, Bajaj JS. Changes in the Microbiome in Cirrhosis and Relationship to Complications: Hepatic Encephalopathy, Spontaneous Bacterial Peritonitis, and Sepsis. Semin Liver Dis. 2016;36:4:327-30.

19. Arab JP, Martin-Mateos RM, Shah VH. Gut-liver axis, cirrhosis and portal hypertension: the chicken and the egg. Hepatol Int. 2018;12(Suppl 1):24-33.

20. Ajibola O, Rowan AD, Ogedengbe CO, Mshelia MB, Cabral DJ, Eze AA, et al. Urogenital schistosomiasis is associated with signatures of microbiome dysbiosis in Nigerian adolescents. Scientific reports. 2019;9 1:829.

21. Jenkins TP, Peachey LE, Ajami NJ, MacDonald AS, Hsieh MH, Brindley PJ, et al. Schistosoma mansoni infection is associated with quantitative and qualitative modifications of the mammalian intestinal microbiota. Sci Rep. 2018;8 1:12072.

22. Floudas A, Aviello G, Schwartz C, Jeffery IB, O'Toole PW, Fallon PG. Schistosoma mansoni Worm Infection Regulates the Intestinal Microbiota and Susceptibility to Colitis. Infect Immun. 2019;87:8.

23. Zhao Y, Yang S, Li B, Li W, Wang J, Chen Z, et al. Alterations of the Mice Gut Microbiome via Schistosoma japonicum Ova-Induced Granuloma. Front Microbiol. 2019;10:352.

24. Kay GL, Millard A, Sergeant MJ, Midzi N, Gwisai R, Mduluza T, et al. Differences in the Faecal Microbiome in Schistosoma haematobium Infected Children vs. Uninfected Children. PLoS Negl Trop Dis. 2015;9 6:e0003861.

25. Schneeberger PHH, Coulibaly JT, Panic G, Daubenberger C, Gueuning M, Frey JE, et al. Investigations on the interplays between Schistosoma mansoni, praziquantel and the gut microbiome. Parasit Vectors. 2018;11 1:168.

26. Wu W, Feng A, Huang Y. Research and control of advanced schistosomiasis japonica in China. Parasitol Res. 2015;114(1):17-27.

27. Song LG, Wu XY, Sacko M, Wu ZD. History of schistosomiasis epidemiology, current status, and challenges in China: on the road to schistosomiasis elimination. Parasitol Res. 2016;115 11:4071- 
81.

28. Cholongitas E, Papatheodoridis GV, Vangeli M, Terreni N, Patch D, Burroughs AK. Systematic review: The model for end-stage liver disease-should it replace Child-Pugh's classification for assessing prognosis in cirrhosis? Aliment Pharmacol Ther. 2005;22(11-12):1079-89.

29. Magoc T, Salzberg SL. FLASH: fast length adjustment of short reads to improve genome assemblies. Bioinformatics. 2011;27 21:2957-63.

30. Edgar RC. UPARSE: highly accurate OTU sequences from microbial amplicon reads. Nat Methods. 2013;10 10:996-8.

31. Edgar RC, Haas BJ, Clemente JC, Quince C, Knight R. UCHIME improves sensitivity and speed of chimera detection. Bioinformatics. 2011;27:16:2194-200.

32. Caporaso JG, Kuczynski J, Stombaugh J, Bittinger K, Bushman FD, Costello EK, et al. QIIME allows analysis of high-throughput community sequencing data. Nat Methods. 2010;7 5:335-6.

33. Edgar RC. Search and clustering orders of magnitude faster than BLAST. Bioinformatics. 2010;26 19:2460-1.

34. Schloss PD, Westcott SL, Ryabin T, Hall JR, Hartmann M, Hollister EB, et al. Introducing mothur: opensource, platform-independent, community-supported software for describing and comparing microbial communities. Appl Environ Microbiol. 2009;75 23:7537-41.

35. Carson JP, Ramm GA, Robinson MW, McManus DP, Gobert GN. Schistosome-Induced Fibrotic Disease: The Role of Hepatic Stellate Cells. Trends Parasitol. 2018;34:6:524-40.

36. Anthony B, Allen JT, Li YS, McManus DP. Hepatic stellate cells and parasite-induced liver fibrosis. Parasit Vectors. 2010;3(1):60.

37. Seki E, Brenner DA. Recent advancement of molecular mechanisms of liver fibrosis. J Hepatobiliary Pancreat Sci. 2015;22 7:512-8.

38. Kamdem SD, Moyou-Somo R, Brombacher F, Nono JK. Host Regulators of Liver Fibrosis During Human Schistosomiasis. Front Immunol. 2018;9:2781.

39. Wei X, Jiang S, Chen Y, Zhao X, Li H, Lin W, et al. Cirrhosis related functionality characteristic of the fecal microbiota as revealed by a metaproteomic approach. BMC Gastroenterol. 2016;16(1):121.

40. Schwimmer JB, Johnson JS, Angeles JE, Behling C, Belt PH, Borecki I, et al. Microbiome Signatures Associated With Steatohepatitis and Moderate to Severe Fibrosis in Children With Nonalcoholic Fatty Liver Disease. Gastroenterology. 2019;157(4):1109-22.

41. Bajaj JS, Thacker LR, Fagan A, White MB, Gavis EA, Hylemon PB, et al. Gut microbial RNA and DNA analysis predicts hospitalizations in cirrhosis. JCI Insight. 2018;3:5.

42. Bajaj JS, Heuman DM, Hylemon PB, Sanyal AJ, White MB, Monteith P, et al. Altered profile of human gut microbiome is associated with cirrhosis and its complications. J Hepatol. 2014;60 5:940-7.

43. Bajaj JS, Betrapally NS, Hylemon PB, Heuman DM, Daita K, White MB, et al. Salivary microbiota reflects changes in gut microbiota in cirrhosis with hepatic encephalopathy. Hepatology. 2015;62(4):1260-71. 
44. Ling Z, Liu X, Cheng Y, Jiang X, Jiang H, Wang Y, et al. Decreased Diversity of the Oral Microbiota of Patients with Hepatitis B Virus-Induced Chronic Liver Disease: A Pilot Project. Sci Rep. 2015;5:17098.

45. Oikonomou T, Papatheodoridis GV, Samarkos M, Goulis I, Cholongitas E. Clinical impact of microbiome in patients with decompensated cirrhosis. World J Gastroenterol. 2018;24 34:3813-20.

46. Bajaj JS, Ahluwalia V, Steinberg JL, Hobgood S, Boling PA, Godschalk M, et al. Elderly patients have an altered gut-brain axis regardless of the presence of cirrhosis. Sci Rep. 2016;6:38481.

47. Zhang L, Wu YN, Chen T, Ren CH, Li X, Liu GX. Relationship between intestinal microbial dysbiosis and primary liver cancer. Hepatobiliary Pancreat Dis Int. 2019;18(2):149-57.

48. Walford RL. THE IMMUNOLOGIC THEORY OF AGING. Gerontologist. 1964;4:195-7.

49. Ferrari TC, Moreira PR. Neuroschistosomiasis: clinical symptoms and pathogenesis. Lancet Neurol. 2011;10:9:853-64.

50. Seki T, Kumagai T, Kwansa-Bentum B, Furushima-Shimogawara R, Anyan WK, Miyazawa Y, et al. Interleukin-4 (IL-4) and IL-13 suppress excessive neutrophil infiltration and hepatocyte damage during acute murine schistosomiasis japonica. Infect Immun. 2012;80(1):159-68.

51. Chen L, Chen Q, Hou W, He L. High-throughput dynamic analysis of differentially expressed genes in splenic dendritic cells from mice infected with Schistosoma japonicum. Immunol Lett. 2017;184:1522.

\section{Tables}

Table 1. Demographic and clinical characteristics of the study population per group. 


\begin{tabular}{|llll|}
\hline & SLC group & Control group & P-value \\
\hline $\mathrm{n}$ & 24 & 25 & \\
\hline Gender (male/female) & $12 / 12$ & $12 / 13$ & 0.889 \\
\hline Age (years) & $82.7 \pm 7.3$ & $82.3 \pm 7.1$ & 0.852 \\
\hline Active smoker (\%) & $0 \%$ & $12 \%$ & 0.248 \\
\hline Active drinker (\%) & $0 \%$ & $20 \%$ & 0.066 \\
\hline BMI (Kg/m $\left.{ }^{2}\right)$ & $22.4 \pm 1.8$ & $23.3 \pm 2.3$ & 0.915 \\
\hline Platelets $\left(10^{9} / \mathrm{L}\right)$ & $152.3 \pm 108.3$ & $179.6 \pm 59.5$ & 0.275 \\
\hline PT (s) & $19.4 \pm 8.1$ & $15.3 \pm 1.6$ & 0.003 \\
\hline Total protein (g/L) & $59.5 \pm 8.3$ & $74.0 \pm 4.1$ & $<0.001$ \\
\hline Albumin (g/L) & $31.4 \pm 5.1$ & $29.6 \pm 4.4$ & 0.186 \\
\hline Globulin $(\mathrm{g} / \mathrm{L})$ & $27.7 \pm 6.0$ & $44.5 \pm 3.8$ & $<0.001$ \\
\hline TB $(\mu \mathrm{mol} / \mathrm{L})$ & $19.8 \pm 19.3$ & $15.3 \pm 5.1$ & 0.711 \\
\hline DB $(\mu \mathrm{mol} / \mathrm{L})$ & $7.9 \pm 9.9$ & $4.2 \pm 1.5$ & 0.509 \\
\hline AST $(\mathrm{U} / \mathrm{L})$ & $32.2 \pm 17.5$ & $17.6 \pm 6.6$ & $<0.001$ \\
\hline ALT $(\mathrm{U} / \mathrm{L})$ & $22.0 \pm 16.0$ & $22.2 \pm 5.4$ & 0.114 \\
\hline GGT $(\mathrm{U} / \mathrm{L})$ & $61.0 \pm 51.6$ & $33.6 \pm 21.5$ & 0.024 \\
\hline Child-Pugh & & & $\mathrm{NA}$ \\
\hline A & 13 & $/$ & $\mathrm{NA}$ \\
\hline B & 7 & $/$ & $\mathrm{NA}$ \\
\hline C & & & \\
\hline
\end{tabular}

Note: The continuous and categorical variables are listed as mean \pm SD and percentage (\%), respectively. Statistical differences were determined using the Student's t-test (parametric data) or the Wilcoxon test (non-parametric data) and the $\chi 2$ test for continuous and categorical variables, respectively. Abbreviations: ALT - alanine aminotransferase; AST - aspartate aminotransferase; BMI - body mass index; DB - direct bilirubin; GGT - glutamyl transpeptidase; PT - prothrombin time; SLC - S. japonicum infectioninduced liver cirrhosis; and TB - total bilirubin. 
Figures

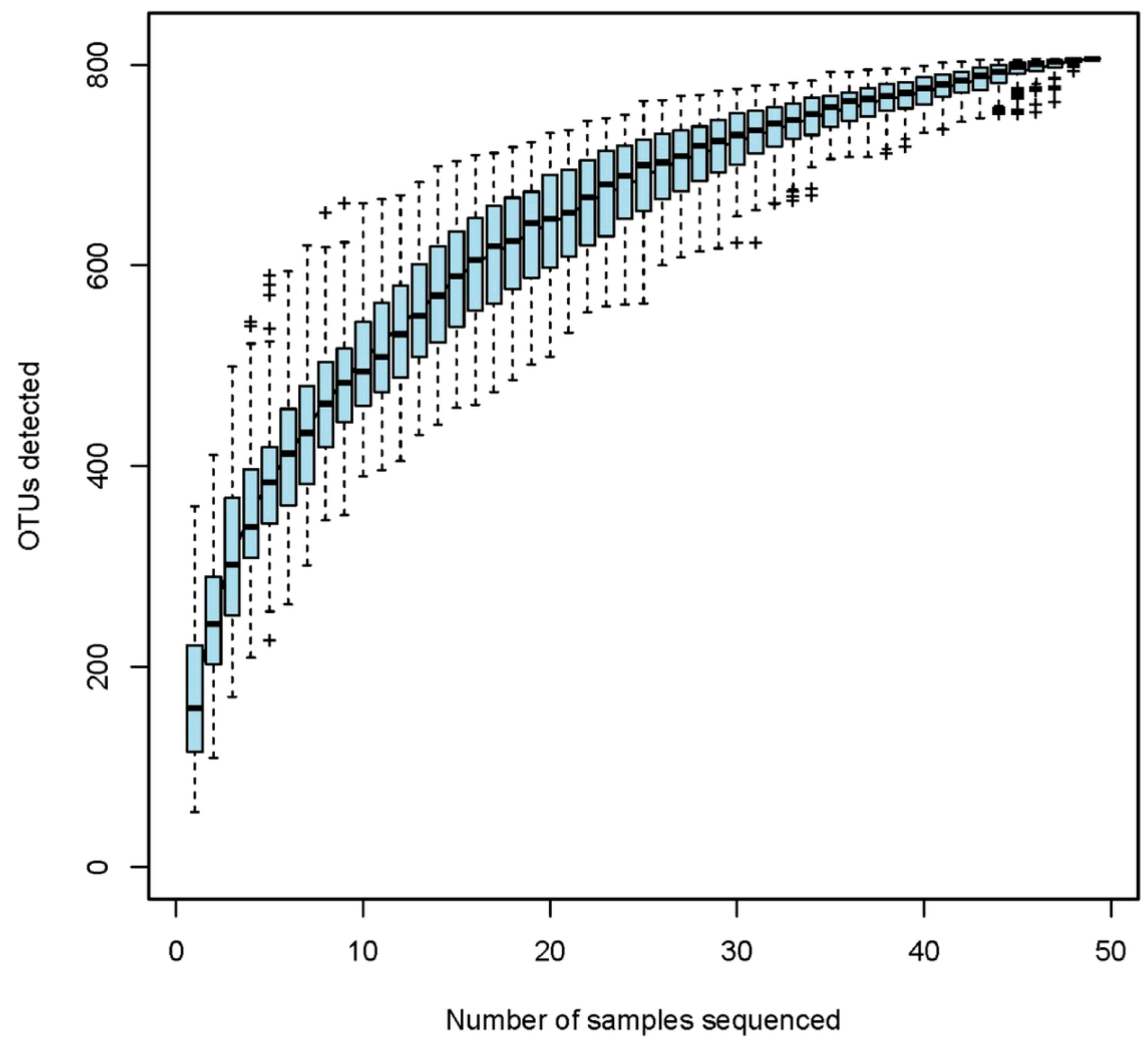

Figure 1

Accumulation curves attest to the sequencing depth adequacy. Accumulation curve is given by cumulative data of OTUs per sample. Each box and whiskers' plot represents OTUs quantified for the 
respective number of samples sequenced (e.g., the first plot refers to one sample, while the second plot represents the cumulative data of two samples, and so forth).

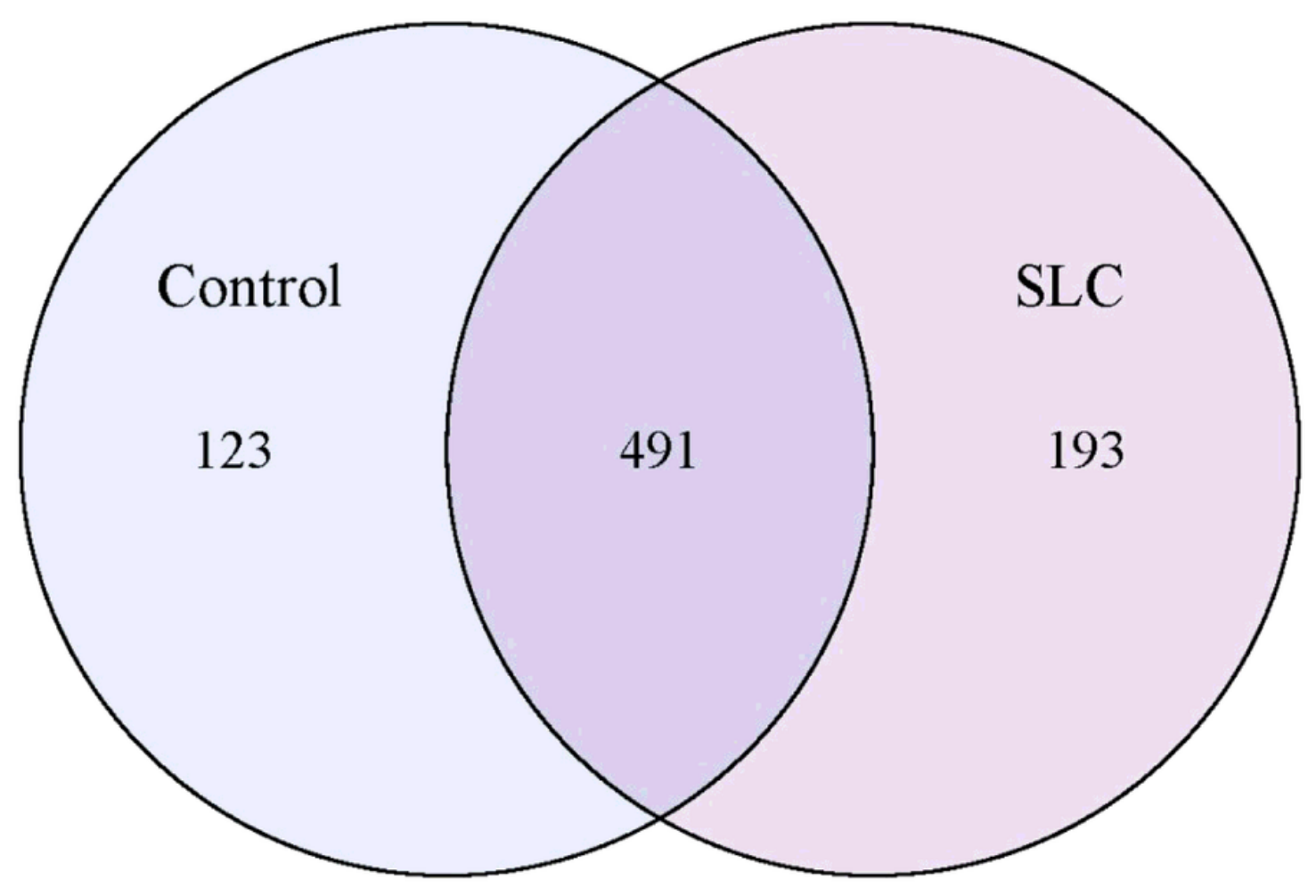

Figure 2

Representation of the OTUs detected per group of samples. Venn diagram depicts the number of OTUs unique for healthy individuals (blue circle) and patients with S. japonicum infection-induced liver cirrhosis (SLC; purple circle), as well as the ones shared by the two groups (circles' intersection). 
A

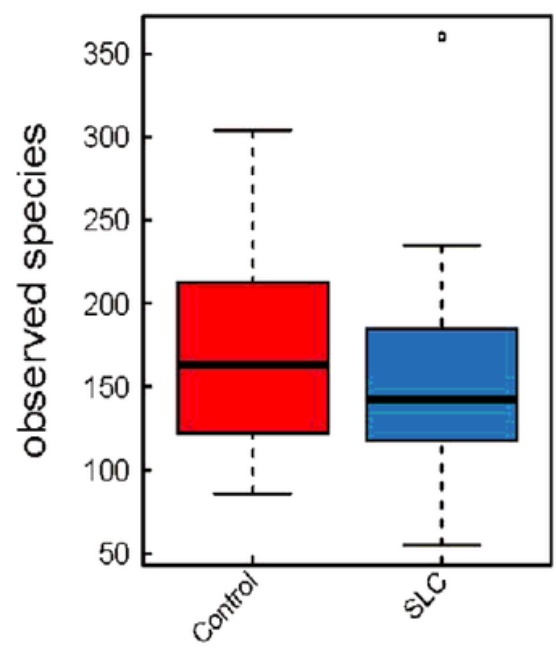

$\mathrm{D}$

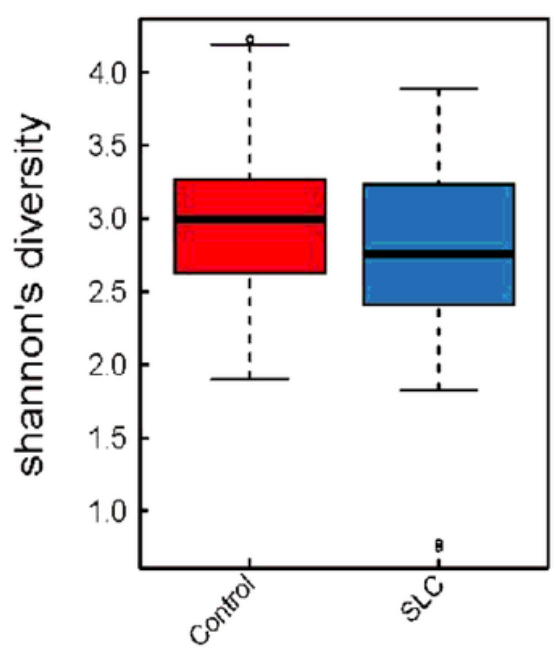

B

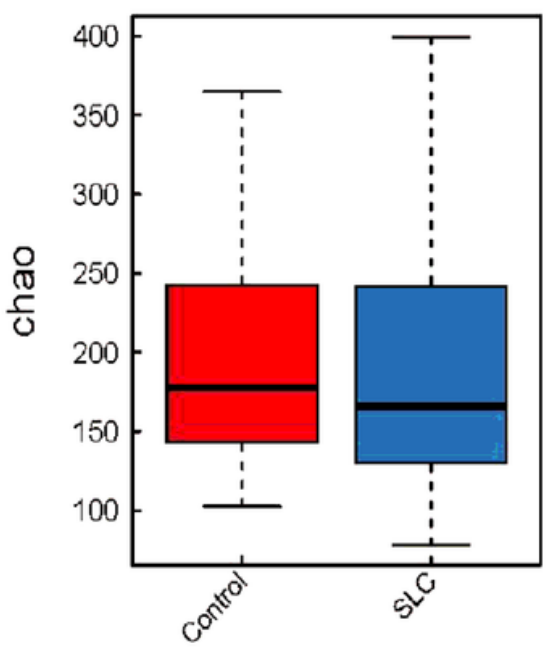

$\mathrm{E}$

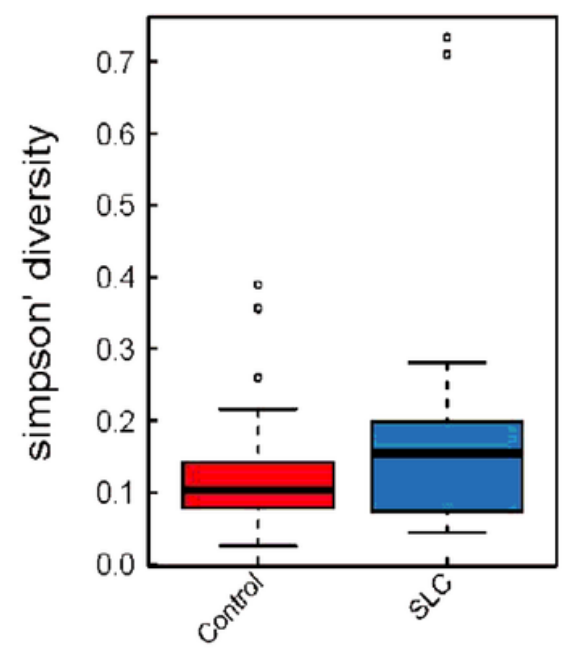

C

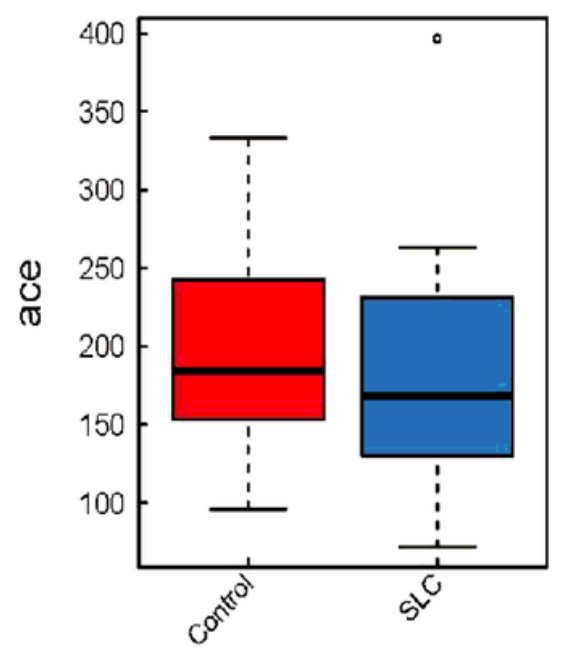

F

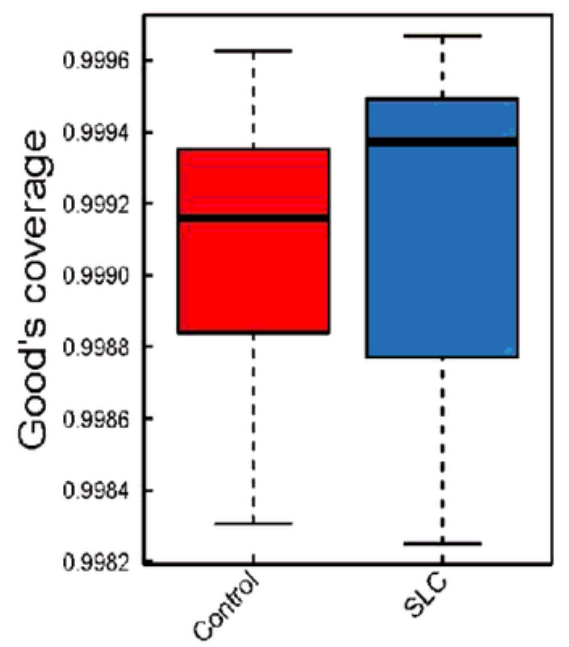

Figure 3

Alpha-diversity estimations are similar for control and cirrhosis groups. Intra-sample bacterial species diversity and richness were evaluated through the calculation of 6 different metrics. Results are presented as box and whiskers' plots per group (control and S. japonicum infection-induced liver cirrhosis - SLC plots in red and blue, respectively) and per metric: (A) Observed species; (B) Chao index; (C) ACE index; (D) Shannon index; (E) Simpson index; and (F) Good-coverage index. No significant differences were detected between groups. 

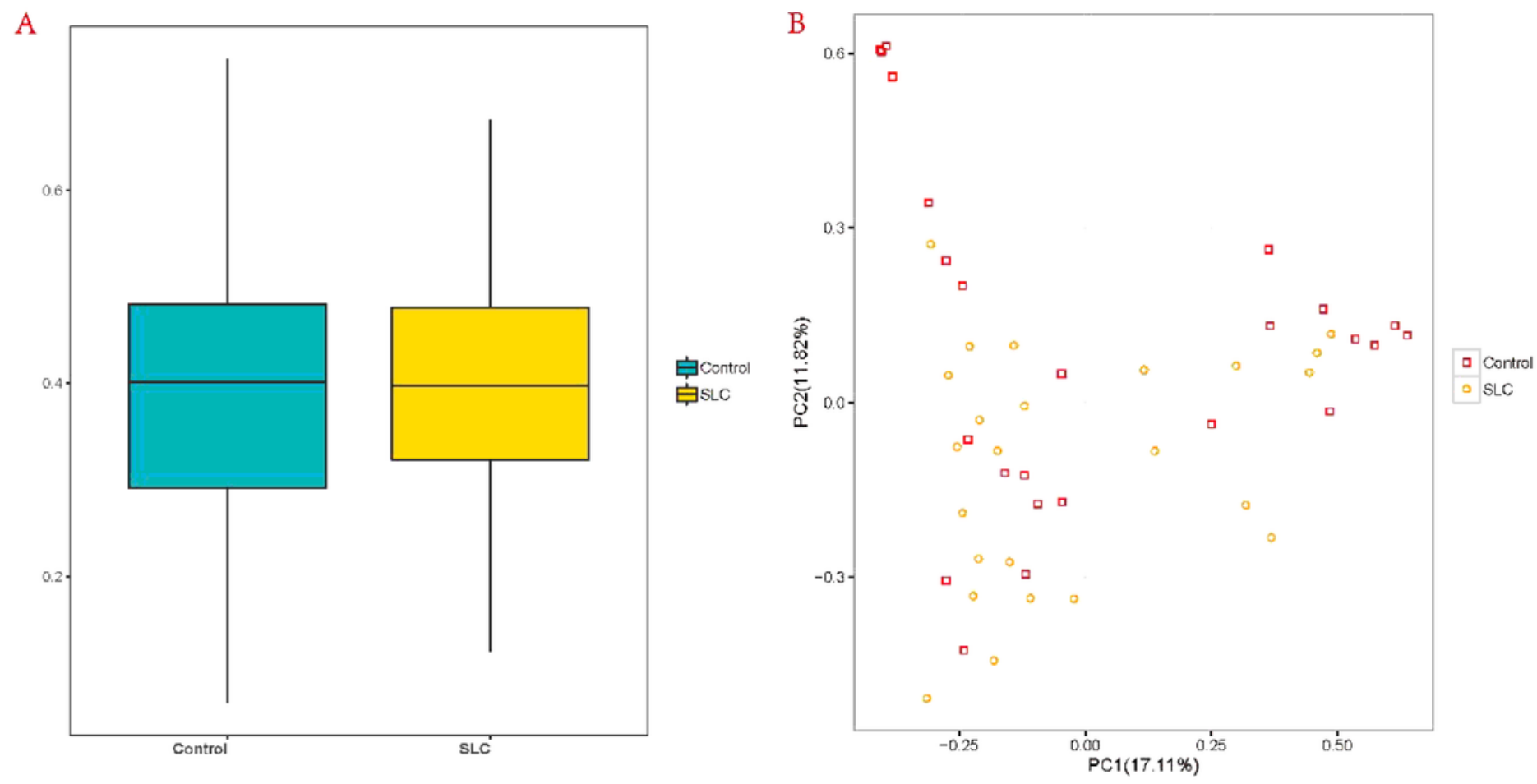

Figure 4

Gut microbiota diversity is not affected in patients with S. japonicum infection-induced liver cirrhosis (SLC). Beta diversity was calculated based on weighted UniFrac distances and is represented per group as box and whiskers' plots (A). A principal component analysis (PCA) was also performed and is represented (per sample, and group) (B). Yellow plot/symbols and blue or red plot/symbols refer to SLC and healthy control samples, respectively. No statistical differences were detected between the two groups. 
A

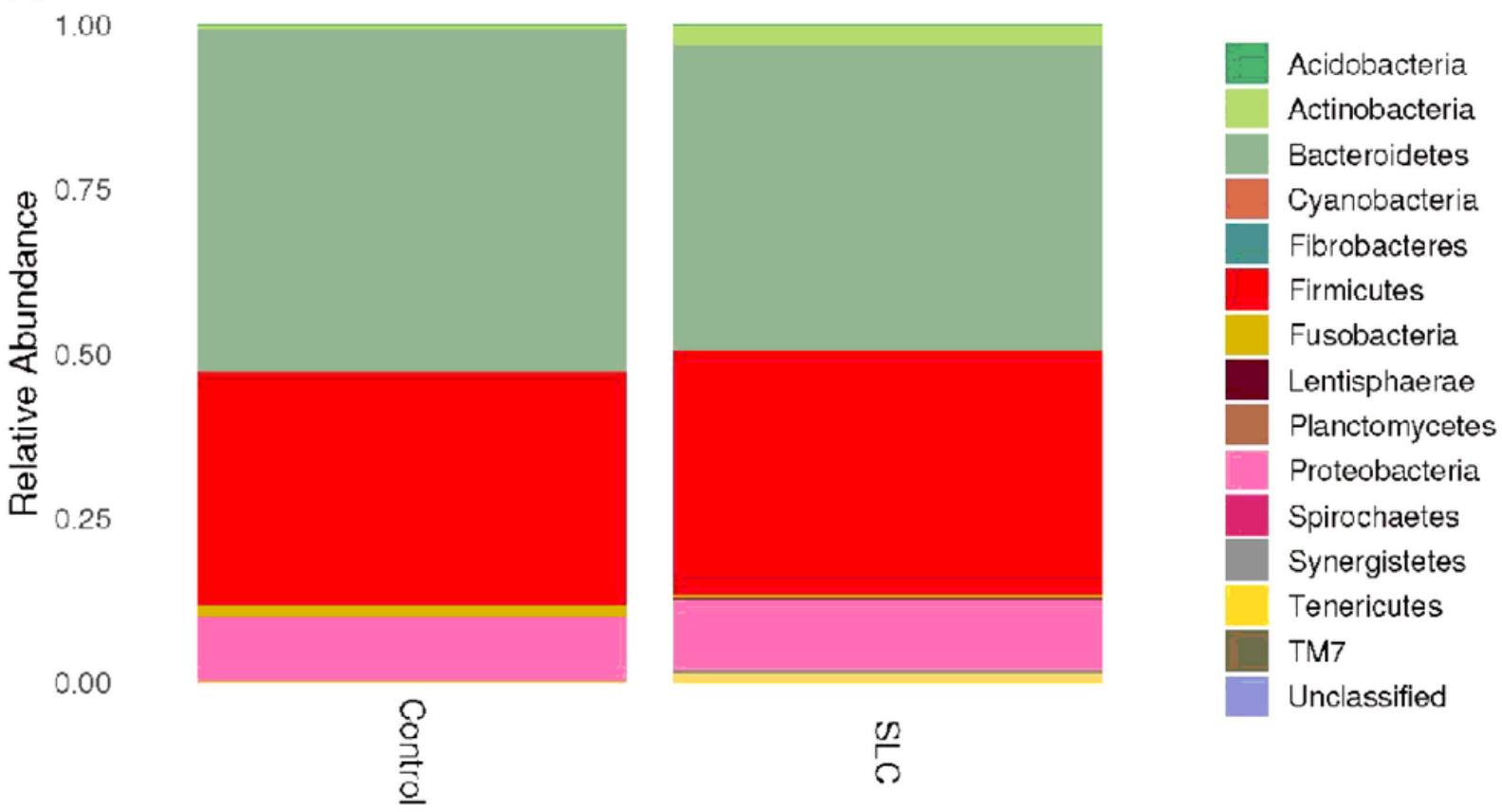

B

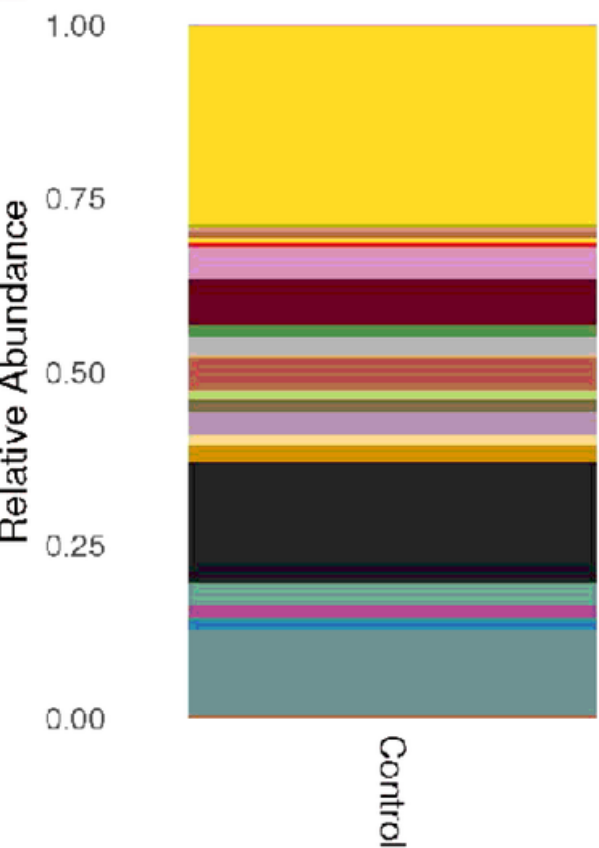

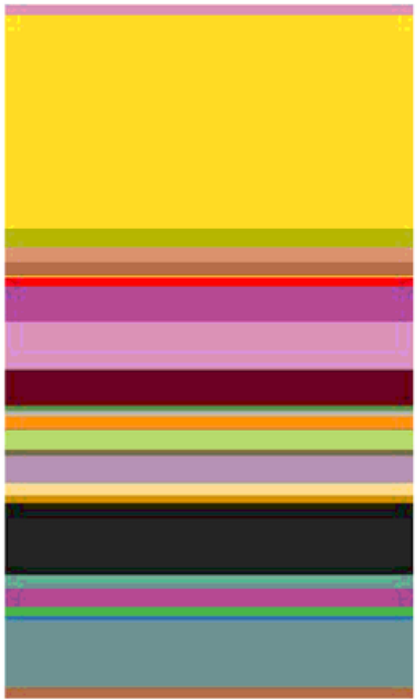

$\frac{\infty}{\circ}$

\begin{tabular}{|l|l|l|}
\hline Akkermansia & Oscillospira \\
\hline Bacteroides & Others \\
\hline Bifidobacterium & Parabacteroides \\
\hline Blautia & Phascolarctobacterium \\
\hline Clostridium & Prevotella \\
\hline Coprococcus & Roseburia \\
\hline Dialister & Ruminococcus \\
\hline Enterococcus & Streptococcus \\
\hline Escherichia & Sutterella \\
\hline Faecalibacterium & Unclassified \\
\hline Fusobacterium & Veillonella \\
\hline Lachnospira & \\
\hline Lactobacillus & \\
Megamonas & \\
Megasphaera & \\
\hline
\end{tabular}

\section{Figure 5}

Gut bacterial composition is affected in S. japonicum infection-induced liver cirrhosis (SLC) patients. The relative abundance of bacterial phyla (A) and genera (B) detected in fecal samples was determined and is represented per group of samples. At the genus level, relative abundances of less than $0.5 \%$ were grouped and are represented as "others." Phyla and genera are color-coded. 


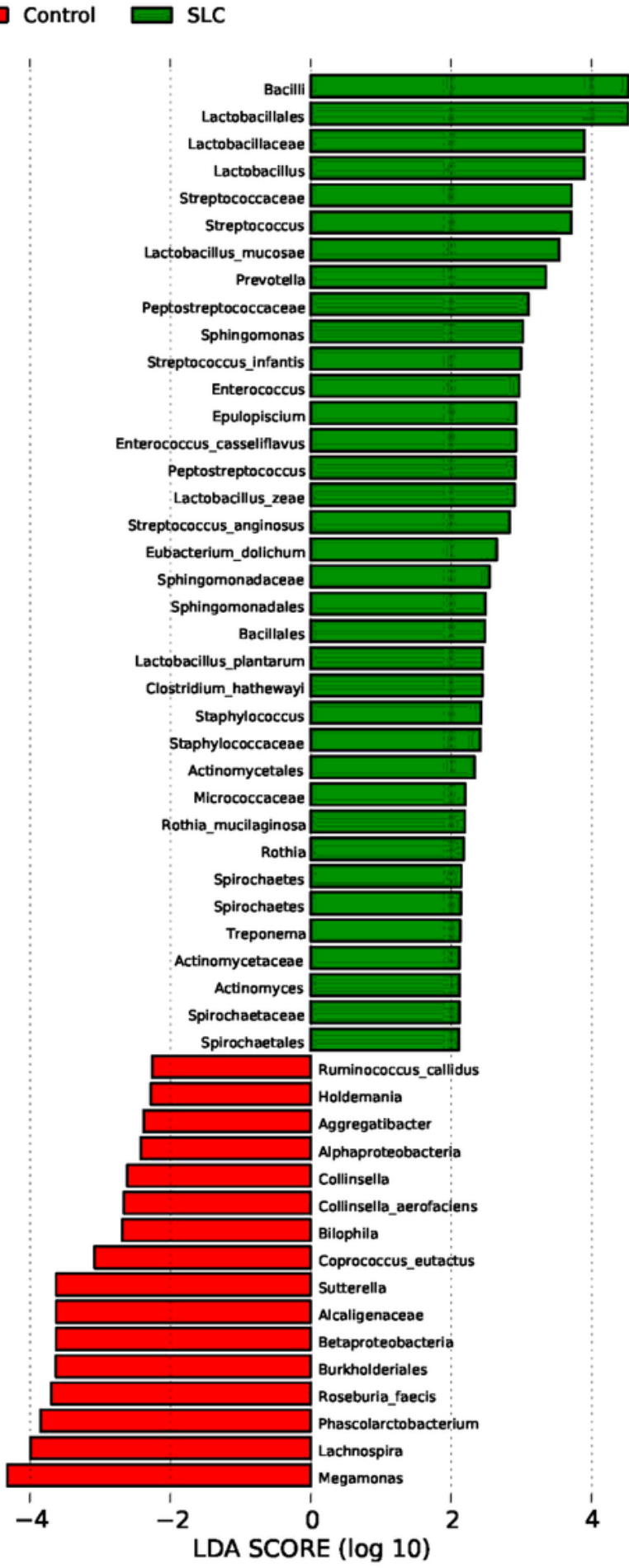

\section{Figure 6}

Taxonomic differences in gut microbiotas of S. japonicum infection-induced liver cirrhosis (SLC) patients. LDA with the calculation of LDA effect size (LEfSe) was performed to identify the differentially abundant taxa in the two groups of samples: represented in red and green for healthy controls and SLC patients, respectively. LDA scores $>2$ were considered statistically significant. 


\section{Cladogram}
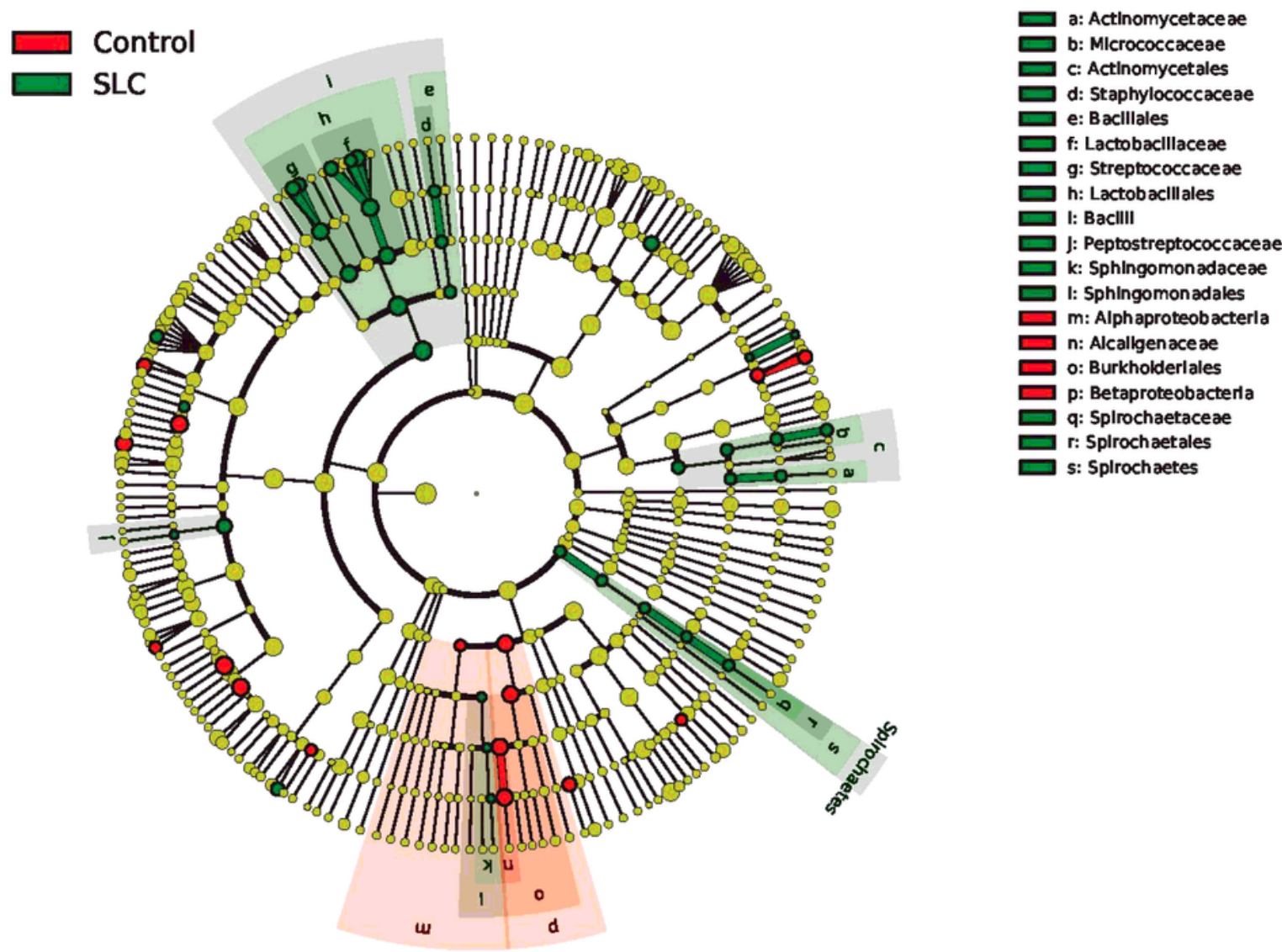

Figure 7

Gut microbiota community differences between healthy individuals and S. japonicum infection-induced liver cirrhosis (SLC) patients. Linear discriminant analysis (LDA) was translated into a cladogram that is represented. The red and green nodes represent specific microorganisms relevant in healthy controls and cirrhosis patients, respectively. The diameter of each node is proportional to the taxon's abundance. Significant bacterial taxonomic groups are identified: genus - g; family - $f$; species $-\mathrm{s}$; order -0 ). 
A

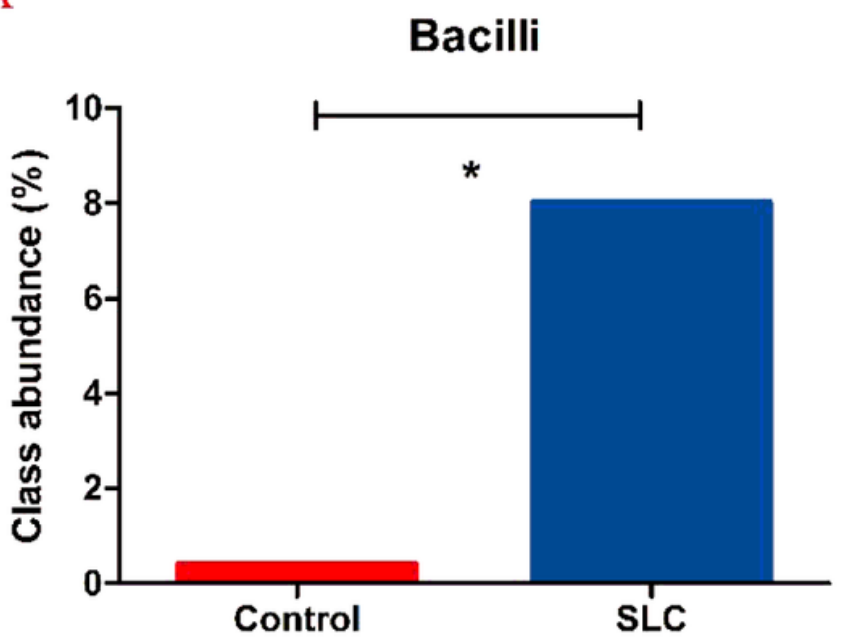

B
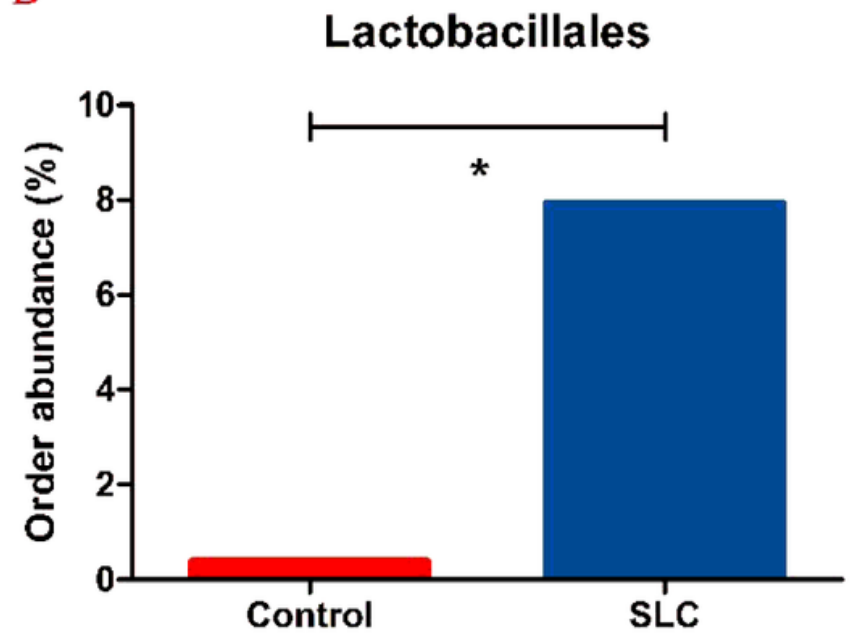

Figure 8

Bacilli Class and Lactobacillales Order are increased in S. japonicum infection-induced liver cirrhosis (SLC) patients. The relative abundance of relevant taxa in the gut of SLC patients at the Class (A) and Order (B) levels was calculated and is represented per group: red and blue columns refer to healthy individuals and SLC patients. Statistical significance was calculated and is represented: * FDR $<0.05$ 